Bull. Korean Math. Soc. 52 (2015), No. 5, pp. 1489-1493

http://dx.doi.org/10.4134/BKMS.2015.52.5.1489

\title{
ON SIMULTANEOUS LOCAL DIMENSION FUNCTIONS OF SUBSETS OF $\mathbb{R}^{d}$
}

\author{
LARS OlSEN
}

\begin{abstract}
For a subset $E \subseteq \mathbb{R}^{d}$ and $x \in \mathbb{R}^{d}$, the local Hausdorff dimension function of $E$ at $x$ and the local packing dimension function of $E$ at $x$ are defined by

$$
\begin{aligned}
& \operatorname{dim}_{\mathrm{H}, \mathrm{loc}}(x, E)=\lim _{r \searrow 0} \operatorname{dim}_{\mathrm{H}}(E \cap B(x, r)), \\
& \operatorname{dim}_{\mathrm{P}, \mathrm{loc}}(x, E)=\lim _{r \searrow 0} \operatorname{dim}_{\mathrm{P}}(E \cap B(x, r)),
\end{aligned}
$$

where $\operatorname{dim}_{H}$ and $\operatorname{dim}_{\mathrm{P}}$ denote the Hausdorff dimension and the packing dimension, respectively. In this note we give a short and simple proof showing that for any pair of continuous functions $f, g: \mathbb{R}^{d} \rightarrow[0, d]$ with $f \leq g$, it is possible to choose a set $E$ that simultaneously has $f$ as its local Hausdorff dimension function and $g$ as its local packing dimension function.
\end{abstract}

\section{Introduction and statement of results}

For a subset $E \subseteq \mathbb{R}^{d}$ and $x \in \mathbb{R}^{d}$, we define the local Hausdorff dimension function of $E$ at $x$ by

$$
\operatorname{dim}_{\mathrm{H}, \mathrm{loc}}(x, E)=\lim _{r \searrow 0} \operatorname{dim}_{\mathrm{H}}(E \cap B(x, r)),
$$

where $\operatorname{dim}_{H}$ denotes the Hausdorff dimension. The local packing dimension function of $E$ at $x$ is defined similarly, i.e., by

$$
\operatorname{dim}_{\mathrm{P}, \operatorname{loc}}(x, E)=\lim _{r \searrow 0} \operatorname{dim}_{\mathrm{P}}(E \cap B(x, r)),
$$

where dimp denotes the packing dimension. The reader is referred to [1] for the definitions of the Hausdorff and the packing dimensions. The local Hausdorff dimension function of a set has recently found several applications in fractal geometry and information theory, cf. $[2,4]$. In [3] we proved that any continuous function is the local Hausdorff dimension function of some set, i.e., if $f: \mathbb{R}^{d} \rightarrow$

Received June 19, 2014

2010 Mathematics Subject Classification. 28A80.

Key words and phrases. Hausdorff dimension, packing dimension, local Hausdorff dimension, local packing dimension. 
$[0, d]$ is continuous, then there exists a set $E \subseteq \mathbb{R}^{d}$ such that

$$
f(x)=\operatorname{dim}_{\mathrm{H}, \mathrm{loc}}(x, E)
$$

for all $x \in \mathbb{R}^{d}$. In this note we give a short and simple proof showing that for any pair of continuous functions $f, g: \mathbb{R}^{d} \rightarrow[0, d]$ with $f \leq g$, it is, in fact, possible to choose the set $E$ such that it simultaneously has $f$ as its local Hausdorff dimension function and $g$ as its local packing dimension function, i.e., such that

$$
\begin{aligned}
& f(x)=\operatorname{dim}_{\mathrm{H}, \text { loc }}(x, E), \\
& g(x)=\operatorname{dim}_{\mathrm{P}, \text { loc }}(x, E),
\end{aligned}
$$

for all $x \in \mathbb{R}^{d}$. In fact, our result also provides information about the rate at which the dimensions $\operatorname{dim}_{\mathrm{H}}(E \cap B(x, r))$ and $\operatorname{dim}_{\mathrm{P}}(E \cap B(x, r))$ converge to $f(x)$ and $g(x)$, respectively, as $r \searrow 0$, see (1.1) below. For an arbitrary function $\varphi: \mathbb{R}^{d} \rightarrow \mathbb{R}$ and $x \in \mathbb{R}^{d}$, we let

$$
\omega_{\varphi}(x, r)=\sup _{x_{1}, x_{2} \in B(x, r)}\left|\varphi\left(x_{1}\right)-\varphi\left(x_{2}\right)\right|
$$

denote the modulus of continuity of $\varphi$ at $x$, and observe that $\varphi$ is continuous at $x$ if and only if $\omega_{\varphi}(x, r) \rightarrow 0$ as $r \searrow 0$.

Theorem 1. Let $f, g: \mathbb{R}^{d} \rightarrow[0, d]$ be continuous functions with $f \leq g$. Then there exists an $\mathcal{F}_{\sigma}$ set $E \subseteq \mathbb{R}^{d}$ such that

$$
\begin{aligned}
& \left|f(x)-\operatorname{dim}_{\mathrm{H}}(E \cap B(x, r))\right| \leq \omega_{f}(x, r), \\
& \left|g(x)-\operatorname{dim}_{\mathrm{P}}(E \cap B(x, r))\right| \leq \omega_{g}(x, r),
\end{aligned}
$$

for all $x \in \mathbb{R}^{d}$ and all $r>0$. In particular,

$$
\begin{aligned}
& f(x)=\operatorname{dim}_{\mathrm{H}, \mathrm{loc}}(x, E), \\
& g(x)=\operatorname{dim}_{\mathrm{P}, \mathrm{loc}}(x, E),
\end{aligned}
$$

for all $x \in \mathbb{R}^{d}$.

\section{Proof of Theorem 1}

In this section we prove Theorem 1. We need the following well-known result in order to prove Theorem 1.

Lemma 2.1. Let $G$ be a non-empty open subset of $\mathbb{R}^{d}$ and $t, s \in \mathbb{R}$ with $0 \leq t \leq s \leq d$. Then there exists a compact set $E \subseteq G$ such that $\operatorname{dim}_{\mathrm{H}}(E)=t$ and $\operatorname{dim}_{\mathrm{P}}(E)=s$.

Proof. For a proof see, for example, [5]. In fact, the result in [5] is formulated and proved for the case where $d=1$, but the techniques in [5] can clearly be adapted to prove the same result in the general case. 
We can now prove Theorem 1. We first introduce some notation. For a function $\varphi: \mathbb{R}^{d} \rightarrow \mathbb{R}$ and $x \in \mathbb{R}^{d}$ and positive number $r>0$, write

$$
\begin{aligned}
& m(\varphi ; x, r)=\inf _{y \in B(x, r)} \varphi(y), \\
& M(\varphi ; x, r)=\sup _{y \in B(x, r)} \varphi(y) .
\end{aligned}
$$

Proof of Theorem 1. Let $0 \leq t<\sup _{x \in \mathbb{R}^{d}} f(x)$ and $0 \leq s<\sup _{x \in \mathbb{R}^{d}} g(x)$ with $t \leq s$ Fix $x \in\{t<f, s<g\}$ and $r>0$. Since $f$ and $g$ are continuous, we conclude that the set $B(x, r) \cap\{t<f, s<g\}$ is open, and it therefore follows from Lemma 2.1 that we can find a compact set $E_{t, s}(x, r)$ satisfying

$$
\begin{aligned}
& E_{t, s}(x, r) \subseteq B(x, r) \cap\{t<f, s<g\}, \\
& \operatorname{dim}_{\mathrm{H}}\left(E_{t, s}(x, r)\right)=t, \\
& \operatorname{dim}_{\mathrm{P}}\left(E_{t, s}(x, r)\right)=s .
\end{aligned}
$$

Next choose a countable dense subset $U_{t, s}$ of $\{t<f, s<g\}$. We now define the set $E$ as

$$
E=\bigcup_{\substack{0 \leq t<\sup _{y \in \mathbb{R}^{d}} f(y) \\ 0 \leq s<\sup _{y \in \mathbb{R}^{d}} \\ t, s \in \mathbb{Q}_{+} \\ t \leq s}} \bigcup_{\substack{r \in \mathbb{Q}_{+} \\ x \in U_{t, s}}} E_{t, s}(x, r)
$$

The set $E$ is clearly $\mathcal{F}_{\sigma}$. We will now prove that $f$ is the local Hausdorff dimension function of $E$ and that $g$ is the local packing dimension function of $E$, i.e., $f(x)=\operatorname{dim}_{\mathrm{H}, \text { loc }}(x, E)$ and $g(x)=\operatorname{dim}_{\mathrm{P}, \text { loc }}(x, E)$ for all $x \in \mathbb{R}^{d}$.

Claim 1. For all $x \in \mathbb{R}^{d}$ and all $r>0$, we have

$$
\begin{aligned}
& \operatorname{dim}_{\mathrm{H}, \mathrm{loc}}(x, E) \leq M(f ; x, r), \\
& \operatorname{dim}_{\mathrm{P}, \mathrm{loc}}(x, E) \leq M(g ; x, r) .
\end{aligned}
$$

Proof of Claim 1. Fix $x \in \mathbb{R}^{d}$ and $r>0$. We now have

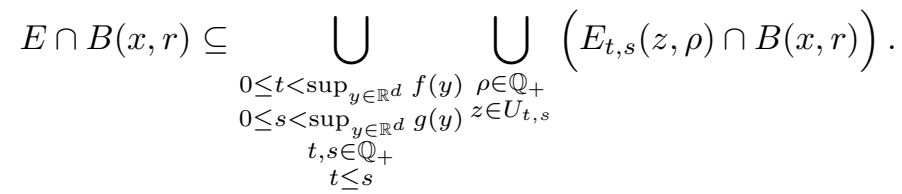

Next observe that since $E_{t, s}(z, \rho) \subseteq\{t<f, s<g\}$, we conclude that

$$
E_{t, s}(z, \rho) \cap B(x, r) \subseteq\{t<f, s<g\} \cap B(x, r)=\emptyset
$$

for $M(f ; x, r) \leq t$ and $M(g ; x, r) \leq s$. Combining (2.1) and (2.2) yields

$$
E \cap B(x, r) \subseteq \bigcup_{\substack{0 \leq t<M(f ; x, r) \\ 0 \leq s<M(g ; x, r) \\ t, s \in \mathbb{Q}_{+} \\ t \leq s}} \bigcup_{\substack{\rho \in \mathbb{Q}_{+} \\ t \in U_{t, s}}}\left(E_{t, s}(z, \rho) \cap B(x, r)\right)
$$




$$
\subseteq \bigcup_{\substack{0 \leq t<M(f ; x, r) \\ 0 \leq s<M(g ; x, r) \\ t, s \in \mathbb{Q}_{+} \\ t \leq s}} \bigcup_{\substack{\rho \in \mathbb{Q}_{+} \\ z \in U_{t, s}}} E_{t, s}(z, \rho) .
$$

Since the union in (2.3) is countable, it follows from (2.3) and the fact that the Hausdorff dimension is countable stable that

$$
\begin{aligned}
& \operatorname{dim}_{\mathrm{H}}(E \cap B(x, r)) \leq \sup _{\substack{0 \leq t<M(f ; x, r) \\
0 \leq s<M(g ; x, r) \\
0 \leq \sup _{+} \\
t, s \in \mathbb{Q}_{+} \\
t \leq s}} \operatorname{dim}_{\mathrm{H}, s}\left(E_{t, s}(z, \rho)\right)
\end{aligned}
$$

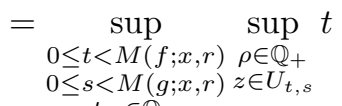

$$
\begin{aligned}
& t, s \in \mathbb{Q}_{+} \\
& =M(f ; x, r)
\end{aligned}
$$

for all $r>0$. Similarly, it follows that

$$
\operatorname{dim}_{\mathrm{P}}(E \cap B(x, r)) \leq M(g ; x, r)
$$

for all $r>0$ This completes the proof of Claim 1 .

Claim 2. For all $x \in \mathbb{R}^{d}$ and all $r>0$, we have

$$
\begin{aligned}
& m(f ; x, r) \leq \operatorname{dim}_{\mathrm{H}, \text { loc }}(x, E), \\
& m(g ; x, r) \leq \operatorname{dim}_{\mathrm{P}, \mathrm{loc}}(x, E) .
\end{aligned}
$$

Proof of Claim 2. Fix $x \in \mathbb{R}^{d}$ and $r>0$. Next, let $\varepsilon>0$ be such that $m(f ; x, r)-\varepsilon, m(g ; x, r)-\varepsilon \in \mathbb{Q}_{+}$. Write $t=m(f ; x, r)-\varepsilon$ and $s=m(g ; x, r)-\varepsilon$, and observe that $t \leq s$. We clearly have $x \in\{t<f, s<g\}$, and we can therefore find $u \in U_{t, s}$ with $|u-x| \leq \frac{r}{2}$. Now, pick any $\rho \in \mathbb{Q}_{+}$with $\rho \leq \frac{r}{2}$. It now follows that

$$
E_{t, s}(u, \rho) \subseteq E,
$$

and that $E_{t, s}(u, \rho) \subseteq B(u, \rho) \subseteq B(x, r)$, whence

$$
E \cap B(x, r) \supseteq E_{t, s}(u, \rho) \cap B(x, r)=E_{t, s}(u, \rho) .
$$

We therefore conclude that

$$
\operatorname{dim}_{\mathrm{H}}(E \cap B(x, r)) \geq \operatorname{dim}_{\mathrm{H}}\left(E_{t, s}(u, \rho)\right)=t \geq m(f ; x, r)-\varepsilon .
$$

Similarly, we conclude that

$$
\operatorname{dim}_{\mathrm{P}}(E \cap B(x, r)) \geq \operatorname{dim}_{\mathrm{P}}\left(E_{t, s}(u, \rho)\right)=s \geq m(g ; x, r)-\varepsilon .
$$

Claim 2 follows from (2.4) and (2.5) by letting $\varepsilon \searrow 0$ through values such that $m(f ; x, r)-\varepsilon, m(g ; x, r)-\varepsilon \in \mathbb{Q}_{+}$.

Theorem 1 follows immediately from Claim 1 and Claim 2. 


\section{References}

[1] K. J. Falconer, Fractal Geometry, John Wiley \& Sons, 1990.

[2] H. Jürgensen and L. Staiger, Local Hausdorff dimension, Acta Inform. 32 (1995), no. 5, 491-507.

[3] L. Olsen, Applications of divergence points to local dimension functions of subsets of $\mathbb{R}^{d}$, Proc. Edinb. Math. Soc. 48 (2005), no. 1, 213-218.

[4] T. Rushing, Hausdorff dimension of wild fractals, Trans. Amer. Math. Soc. 334 (1992), no. 2, 597-613

[5] D. Spear, Sets with different dimensions in [0,1], Real Anal. Exchange 24 (1998/99), no. 1, 373-389.

Department of Mathematics

University of St. Andrews

St. Andrews, Fife KY16 9SS, Scotland

E-mail address: lo@st-and.ac.uk 